\title{
Análisis de una serie de 55 pacientes con arteritis de células gigantes confirmada por biopsia
}

\author{
E. BUSTAMANTE MALDONADO, B. MARÍ ALFONSO, M. MONTEAGUDO \\ JIMÉNEZ, A. CASANOVAS MARTÍNEZ, R. JORDANA COMAJUNCOSA, \\ C. TOLOSA VILELLA, J. ORISTRELL SALVÁ \\ Servicio de Medicina Interna. Corporació Sanitària Parc Taulí. Instituto Universitario \\ Parc Taulí. UAB. Sabadell. Barcelona
}

\section{RESUMEN}

Introducción: Descripción de las características epidemiológicas, clínicas, respuesta al tratamiento y complicaciones de una serie de 55 pacientes con arteritis de células gigantes(ACG) confirmada por biopsia en un hospital comarcal.

Material y métodos: Estudio retrospectivo de los pacientes diagnosticados de ACG por biopsia de arteria temporal en el periodo comprendido entre 1989 y 2001 en nuestro centro.

Resultados: La incidencia anual aproximada calculada en nuestra área sería de 4,1 casos por 100.000 habitantes mayor de 50 años. La edad media al diagnóstico fue de 74 años y un $78,2 \%$ eran mujeres. El síntoma más frecuente al diagnóstico fue la cefalea $(81,5 \%)$, seguido de las manifestaciones sistémicas $(74,1 \%)$, a más distancia se encontraba la claudicación mandibular $(32,7 \%)$, trastornos visuales $(30,2 \%)$, manifestaciones isquémicas (17\%). La polimialgia reumática se asoció a ACG en el $49,1 \%$. La palpación de arteria temporal fue anormal en el 76,9\% de los pacientes. La dosis media de inicio de tratamiento fue de $69 \mathrm{mg}$ prednisona /dia, con una media de tiempo de reducción a mitad de dosis fue de 3,5 meses. Un $24,1 \%$ de los pacientes presentaron un rebrote durante el primer año. Un $38 \%$ de los pacientes presentaron complicaciones durante el tratamiento corticoideo. La VSG fue inferior a $50 \mathrm{~mm}$ en el $12,7 \%$ de los pacientes, existía anemia en el 37,7\% y trombocitosis en el 32,1\%. En estos últimos se detectó una tendencia a la relación con presencia de alteraciones visuales específicas.

Conclusiones: La incidencia de ACG en nuestra área estaría dentro de las zonas de baja incidencia. Los resultados de nuestra serie no difieren de otras descritas previamente en la literatura en cuanto a las manifestaciones clínicas de los pacientes, existe un claro predominio del sexo femenino y se detecta una tendencia a la relación de alteraciones visuales específicas con presencia de trombocitosis.

PALABRAS CLAVE: Arteritis temporal. Arteritis de células gigantes. Enfermedad de Horton. Vasculitis. Biopsia de arteria temporal. ANALYSIS OF A SERIE OF 55 PATIENTS WITH BIOPSY PROVEN
GIANT CELL TEMPORAL ARTERITIS

\begin{abstract}
Introduction: Our main aim with this study is to establish the epidemiologic and clinical features, treatment response and complications of a group composed of 55 patients with biopsy proven temporal arteritis in a local hospital.

Material and methods: Restrospective study based on clinical records revision of patients diagnosticated of giant cell arteritis (GCA) made by temporal artery biopsy between 1989 and 2001.

Results: The aproximated annual incidence of GCA in our area is 4.1 cases per 100.000 persons over the age of 50. The mean age at diagnostic was 74 years and the 78,2\% were women. The most common symptom at diagnostic was headache (81,5\%) followed by systemic manifestations $(74,1 \%)$ and later we found jaw claudication $(32,7 \%)$, visual impairment $(30,2 \%)$, isquemic manifestations (17\%). Polimyalgia rheumatica was asociated to GCA in 49,1\% of cases. The temporal artery explorations was abnormal in the $76,9 \%$ of patients. The mean initial dose of corticoids was $69 \mathrm{mg}$ prednisone per day, with a half dose reduction time of 3,5 months. In spite of that, $24,1 \%$ of patients relapsed during the first year. The $38 \%$ of patients did some complications during the corticosteroid treatment. The ESR was lower $50 \mathrm{~mm}$ in $12,7 \%$ of patients; it was anaemia in the $37,7 \%$ and thrombocitosis in $32,1 \%$. In these last patients we detected a relation between thrombocitosis and specific visual impairment.

Conclusions: The incidence of GCA in our area is low. The results of our serie aren't different from others publicated before in clinical manifestations, there is a prevalence of female sex and there is a relation between specific visual impairment and the presence of thrombocitosis.
\end{abstract}

KEY WORDS: Temporal arteritis. Giant cell arteritis. Horton disease. Vasculitis. Temporal artery biopsy.

Bustamante Maldonado E, Marí Alfonso B, Monteagudo Jiménez, M, Casanovas Martínez A, Jordana Comajuncosa R, Tolosa Vilella C, Oristrell Salvá J. Análisis de una serie de 55 pacientes con arteritis de células gigantes confirmada por biopsia. An Med Interna (Madrid) 2004; 21: $473-476$. 


\section{INTRODUCCIÓN}

La arteritis de células gigantes (ACG) es una vasculitis de etiología desconocida que afecta vasos de grande y mediano calibre en pacientes mayores de 50 años. Característicamente afecta aorta y sus ramas proximales $(1,2)$, con predilección por las arterias extracraneales, aunque se pueden afectar arterias en cualquier otra zona del organismo. Su diagnóstico se basa inicialmente en la sospecha clínica, cuando aparecen los síntomas clásicos de afectación craneal (cefalea, claudicación mandibular, alteraciones visuales, alteraciones en la palpación de arteria temporal) o afectación sistémica (fiebre, anorexia, astenia, polimialgia reumática). Hasta en el $40 \%$ de pacientes la presentación sería con síntomas atípicos (3) y en algunas series la frecuencia de los síntomas clásicos es cada vez menor (4). El diagnóstico de seguridad sigue siendo la presencia de alteraciones anatomopatológicas compatibles en la biopsia de arteria temporal, aunque por la característica de afectación segmentaria de la enfermedad, ésta no siempre es diagnóstica. En 1990 The American College of Rheumatology (ARA) formuló una serie de criterios de clasificación (5) para la ACG con una alta sensibilidad y especificidad cuando existe un diagnóstico previo de vasculitis.

Con nuestro estudio queremos establecer las características epidemiológicas, clínicas, la respuesta al tratamiento y las complicaciones del mismo en una serie de 55 pacientes con arteritis de células gigantes demostrada por biopsia.

\section{MATERIAL Y MÉTODOS}

Datos epidemiológicos. El hospital Parc Tauli de Sabadell es un hospital de Nivel II con 450 camas de agudos, con una poblacion de referencia de 381.000 habitantes, pero con una penetración real en el área del $60 \%$, dada la ubicación del hospital y la presencia de hospitales cercanos en el área del Vallés y en Barcelona. La población de 50 años o superior es de 119.132 (31,26\%) (mujeres: 64.820 varones: 54.312) según padrón municipal del 2000.

Criterios de inclusión. Se incluyeron todos aquellos pacientes a los cuales se les realizó una biopsia de arteria temporal por sospecha de ACG entre enero de 1989 y diciembre de 2001 de los cuales se disponía de datos suficientes en su historia clínica. Se diagnosticó de ACG positiva cuando los hallazgos histológicos de la arteria temporal demostraron ruptura de la lámina elástica interna con infiltrado inflamatorio de la pared arterial con o sin presencia de células gigantes. Se recogieron de forma retrospectiva, a partir de una hoja de datos común una serie de variables: epidemiológicas: edad, sexo, ciudad de residencia. Clínicas: síntomas de debut, tiempo de evolución, cefalea, claudicación mandibular, polimialgia reumática, síntomas sistémicos, alteraciones visuales, manifestaciones isquémicas. Exploración física: palpación de arteria temporal. Datos analíticos: previos al inicio de tratamiento corticoideo y los más cercanos a la fecha de la biopsia. Tratamiento corticoideo: fecha de inicio, dosis de inicio, respuesta al tratamiento, tiempo de reducción a mitad de dosis, rebrotes, dosis con los rebrotes, complicaciones.

Definiciones clínicas. Las variables clínicas se consideraron positivas o negativas sólo si así se reflejaba en la historia clínica y ausente cuando ésta no estaba recogida.

1. Cefalea. Se consideró cuando ésta era de reciente aparición y era un síntoma relevante en la clínica del paciente.
2. Polimialgia reumática. Cuando los pacientes asociaban a la sintomatología propia de arteritis temporal clínica de dolor y/o debilidad en cinturas escapular y/o pelviana.

3. Síntomas sistémicos. Consideramos la presencia de fiebre, astenia, anorexia, pérdida de peso o asociación de varios de ellos.

4. Clínica de debut. Sintomatología inicial y que motivaba la consulta de los pacientes.

5. Alteraciones visuales. Presencia o ausencia de amaurosis fugax, amaurosis permanente, disminución de agudeza visual, fotopsias, miodesopsias, diplopia. Se consideraron alteraciones visuales específicas; amaurosis fugax, amaurosis permanente y diplopia; alteraciones inespecíficas el resto.

6. Palpación de arteria temporal. Se diferenció entre palpación normal o anormal. Se consideró alteración inespecifica cuando existía sólo asimetría de pulso. Se consideró específica cuando existía ausencia de pulso, engrosamiento, dolor a la palpación, nódulos en cuero cabelludo o signos inflamatorios.

7. Rebrote: reaparición de clínica con o sin alteraciones analíticas que obliga a aumentar dosis de corticoides.

Análisis estadístico. Se creó una base de datos con las diferentes variables. Para el análisis de las variables categóricas se realizó el test de chi-cuadrado. Cuando el valor esperado mínimo era menor a 5 se aplicó el test exacto de Fisher. Los cálculos se realizaron con el paquete estadístico SPSS 9.0 para Windows.

\section{RESULTADOS}

Se realizaron 195 biopsias de arteria temporal (BAT), de las cuales 55 fueron positivas para arteritis de células gigantes (ACG) $(28,2 \%)$. Cinco de los pacientes eran anteriores a 1989 y 50 entre 1989-2001. La edad media al diagnóstico fue de 74 años (59-88), siendo mujeres 43 de ellas(78,2\%).

La incidencia de casos nuevos en el año 2000 fue de 3 en una población de 119.132 habitantes mayores de 50 años, con una penetrancia del hospital en la población del $60 \%$ (71.479) nos daría una incidencia aproximada de 4,2 x $10^{5}$ mayores de 50 años. El promedio anual de nuevos diagnósticos fue de 4; máximo 8 en 1997 y mínimo 0 en 1992 y 1999 (Tabla I).

Las manifestaciones clínicas, resumidas en la tabla II, fueron cefalea en 44/54 (81,5\%), claudicación mandibular en $17 / 52(32,7 \%)$, se vio asociación con polimialgia reumática en 26/53 (49,1\%), presencia de síntomas sistémicos en 40/54 $(74,1 \%)$, trastornos visuales en 16/53 (30,2\%) de los cuales 10 tenian alteraciones visuales específicas, manifestaciones isquémicas en 9/53 (17\%). Respecto a la palpación de arteria temporal, fue anormal en 40/52 pacientes $(76,9 \%)$, siendo ésta específica en 26 pacientes y no específica en 14. 54 de los pacientes recibieron tratamiento corticoideo, siendo la dosis media de inicio $69 \mathrm{mg}$ de prednisona al día (20-1000 mg). El tiempo de reducción a mitad de dosis fue de 3,5 meses $(0,3-9)$. Se detectaron 13 rebrotes durante el primer año solo en 1 caso con $20 \mathrm{mg}$ de prednisona al día. El número de rebrotes tratados durante el seguimiento fue de 21/54 (38,9\%). Veintiuno $(38,9 \%)$ de los pacientes presentaron una o mas complicaciones en el curso del tratamiento: aplastamientos vertebrales (8), cataratas (5), infecciones (2), diabetes mellitus (2), infarto de miocardio (2), amiloidosis secundaria (1), otras complicaciones (pancreatitis, hemorragia digestiva, necrosis aséptica femoral y escafoides, migraña esteroidea) (Tabla III). 
TABLA I

BIOPSIAS REALIZADAS Y BIOPSIAS POSITIVAS ENTRE 1989-2001

\begin{tabular}{lccc}
\hline Año & $\begin{array}{c}\text { Número } \\
\text { de BAT }\end{array}$ & $\begin{array}{c}N^{\circ} \text { de } \\
\text { BAT positivas }\end{array}$ & $\begin{array}{c}\% \text { de biopsias } \\
\text { positivas }\end{array}$ \\
\hline$<1988$ & & 5 & \\
1989 & 9 & 7 & $78 \%$ \\
1990 & 20 & 4 & $20 \%$ \\
1991 & 20 & 4 & $20 \%$ \\
1992 & 19 & 0 & $0 \%$ \\
1993 & 15 & 7 & $47 \%$ \\
1994 & 10 & 3 & $30 \%$ \\
1995 & 13 & 2 & $15 \%$ \\
1996 & 9 & 4 & $44 \%$ \\
1997 & 22 & 8 & $37 \%$ \\
1998 & 16 & 3 & $19 \%$ \\
1999 & 6 & 0 & $0 \%$ \\
2000 & 12 & 3 & $25 \%$ \\
2001 & 18 & 5 & $28 \%$ \\
\hline
\end{tabular}

TABLA II

\begin{tabular}{lcr}
\multicolumn{3}{c}{ MANIFESTACIONES CLÍNICAS } \\
\hline Cefalea & $44 / 54(81,5 \%)$ & \\
Síntomas sistémicos & $40 / 54(74,1 \%)$ & \\
Polimialgia reumática & $26 / 53(49,1 \%)$ & \\
Claudicación mandibular & $17 / 52(32,7 \%)$ & \\
Trastornos visuales & $16 / 53(30,2 \%)$ & específico 10/16 \\
& & inespecífico 6/16 \\
Manifestaciones isquémicas & $9 / 53(17 \%)$ & \\
Palpación A. temporal anormal & $40 / 52(76,9 \%)$ & \\
Complicaciones & $21 / 54(38.9 \%)$ \\
Éxitus & $7 / 54(12,9 \%)$ & \\
\hline
\end{tabular}

\section{TABLA III}

\section{COMPLICACIONES DURANTE EL TRATAMIENTO}

Aplastamientos vertebrales

Cataratas
D. mellitus
Infarto miocardio
Otras (1)
Amiloidosis secundaria
Pancreatitis
Hemorragia digestiva
Necrosis aséptica femoral
Necrosis aséptica escafoides
Migraña esteroidea

Respecto a los datos analíticos, la VSG fue superior a 50 $\mathrm{mm}$ en $48(87,3 \%)$ e inferior a $50 \mathrm{~mm}$ en $7(12,7 \%)$, la anemia, definida como hemoglobina inferior o igual a $10 \mathrm{mg} / \mathrm{dl}$, fue detectada en el 37,7\%. 17/53 (32,1\%) de los pacientes presentaron trombocitosis superior a $400 \times 10^{9}$ plaquetes $/ \mathrm{L}, \mathrm{y}$ existía en estos pacientes una tendencia a la presencia de alteraciones visuales específicas, aunque el número de pacientes es pequeño $(p=0,058)$. Siete de los pacientes diagnosticados de ACG fallecieron: 2 por cardiopatía isquémica, 2 insuficiencia renal o complicaciones relacionadas, linfoma, insuficiencia respiratoria, isquemia intestinal masiva.

\section{DISCUSIÓN}

La incidencia de $4,2 \times 10^{5}$ habitantes mayores de 50 años en nuestra área solo puede ser un cálculo aproximado derivado de la inferencia que nuestro centro solo ha atendido al $60 \%$ de la población de referencia en los años analizados. Esta incidencia nos situaría dentro de la zona de incidencia baja (7-9), sin embargo el progresivo envejecimiento de la población puede hacer que el número real de nuevos diagnósticos aumente claramente. Las oscilaciones en el número de biopsias positivas anuales no tiene relación con el número de biopsias realizadas e iría a favor de una posible evolución cíclica de la enfermedad como algunos autores han descrito (10-12) .

Respecto a la distribución por sexos, apreciamos una relación 3/1 en el sexo femenino respecto a masculino, que coincide con la relación hallada en otras series (13-15), mientras que difiere de otras (16-18), estas últimas series publicadas en nuestro país.

En nuestra serie las manifestaciones clínicas en orden decreciente de frecuencia fueron cefalea, presencia de síntomas sistémicos, asociación con polimialgia reumática, claudicación mandibular, trastornos visuales, presencia de manifestaciones isquémicas. Sin grandes diferencias respecto a otras series publicadas en nuestro país, en la serie de Armona et al. (16) la frecuencia de síntomas clínicos fueron cefalea $(87,4 \%)$, polimialgia reumática $(49,2 \%)$, claudicación mandibular $(39,8 \%)$, fiebre $(28,7 \%)$, mientras en la serie de Villalta (17) mostró síndrome tóxico $(79 \%)$, cefalea $(74 \%)$, fiebre $(60 \%)$, mialgias $(30 \%)$, trastornos visuales $(23 \%)$, claudicación mandibular (23\%), polimialgia reumática (19\%).

En la ACG las alteraciones visuales pueden llevar a la ceguera permanente e irreversible en el $10-20 \%$ de paciente (19-22). En nuestra serie el 30,2\% de los pacientes presentaron trastornos visuales, siendo éstos específicos (amaurosis fúgax, permanente, diplopía) en el $62,5 \%$, mientras que en el $37,5 \%$ fueron inespecíficos. El 18,8\% de los pacientes que presentaron trastornos visuales lo hicieron en forma de ceguera permanente.

La ACG es una vasculitis sistémica que afecta predominantemente las arterias extracraneales pero puede afectar cualquier arteria elástica de mediano o gran calibre. Nuestros pacientes presentaron manifestaciones isquémicas a otros niveles en el 17,3\% (9/52), siendo en 6 casos cerebrales, 2 coronario y 1 digestivo, la mayoría al diagnóstico o al inicio del tratamiento.

Las alteraciones analíticas que se pueden encontrar en la ACG son de carácter inespecífico, en relación al proceso inflamatorio. Es común la presencia de una velocidad de sedimentación globular elevada, con frecuencia superior a 100 $\mathrm{mm} / \mathrm{h}$ junto otras alteraciones como son la presencia de anemia y trombocitosis, o un aumento en las cifras de fosfatas alcalina, alfa-2-globulina y proteina $\mathrm{C}$-reactiva habiendo sido comunicada la asociacion inversa entre respuesta inflamatoria y riesgo visual (20). En nuestra serie el 13\% de los pacientes 
presentaron una VSG inferior a $50 \mathrm{~mm} / \mathrm{h}$ y la anemia se presentó en el $37 \%$ de los enfermos. Es destacable la tendencia a mayor alteraciones visuales específicas en los pacientes con trombocitosis $(32,1 \%)$, como ha sido descrito por otros autores (23), aunque dado el escaso número de pacientes las diferencias no son estadisticamente significativas.

El tratamiento habitual es con corticoides a dosis iniciales de 40-60 mg/d (1,2,21). Cuando existe afectación ocular en forma de pérdida de visión se utilizan altas dosis de corticoides endovenosos, no obstante, una vez establecida la amaurosis la recuperación de la visión es inhabitual $(20,21)$. El objetivo del tratamiento es usar la mínima dosis de esteroides durante el período mas corto posible de tiempo controlando adecuadamente los síntomas (1), desafortunadamente entre $30-50 \%$ de pacientes presentan exacerbaciones espontaneas de la enfermedad independientemente del régimen de tratamiento, especialmente durante los primeros 2 años (22). El tratamiento prolongado con corticoides, dará lugar a efectos secundarios y complicaciones que pueden ser importantes. Entre las complicaciones mas frecuentes, como en nuestra serie las fracturas osteoporóticas, por lo que el tratamiento profiláctico con calcio, vitamina $\mathrm{D}$ y difosfonatos en los pacientes con osteopenia es obligado $(24,25)$. Es por tanto objetivo prioritario en la actualidad la búsqueda nuevos tratamientos o pautas alternativas que disminuyan la morbilidad en estos pacientes.

\section{Bibliografía}

1. Evans JM, Hunder GG. Polymyalgia rheumatica and giant cell arteritis. Clin in Geriatr Med 1998; 14: 455-73.

2. Calvo Romero JM. Arteritis de células gigantes. An Med Interna (Madrid) 2002; 19: 257-262.

3. Levine SM, Hellmann DB. Giant cell arteritis.Curr Opin Rheumatol 2002; 14: 3-10.

4. González-Gay MA, Blanco R, Sánchez-Andrade A, Vázquez-Caruncho M. Giant cell arteritis in Lugo, Spain: A more frecuent disease with fewer classic features. J Rheumatol 1997; 24: 2166-80.

5. Hunder GG, Bloch DA, Michel BA, Stevens MB, Arend WP, Calabrese LH et al. The American College of Rheumatology 1990 criteria for the clasification of giant cell arteritis. Arthritis Rheum 1990; 33: 11221128.

6. Lie JT, Members and Consultants of the American College of Rheumatology Subcommittee on Classification of Vasculitis: Illustrated histopathology classification criteria for selectec vasculitis syndrome. Arthritis Rheum 1990; 33: 1074-87.

7. Salvarani C, Macchioni P, Zizzi F, Mantovani W, Rossi F, Castri C, et al. Epidemiologic and immunogenetic aspects of polymyalgia rheumatica and giant cell arteritis in northem Italy (see comments) Arthritis Rheum 1991; 34: 351-6

8. Sonnenblick M, Nesher G, Friedlander Y, Rubinow A. Giant cell arteritis in Jerusalem: a 12-year epidemiological study. Br J Rheumatol 1994; 33: 998-41.

9. Smith CA, Fidler WJ, Pinals RS. The epidemiology of giant cell arteritis. Report of a ten-year study in Shelby County, Tennessee. Arthritis Rheum 1983; 26: 1214-9

10. Elling P, Olsson AT, Elling H. Synchronous variations of incidence of temporal arteritis and polymyalgia rheumatica in different regions of Denmark; association with epidemics of mycoplasma pneumoniae infection. J Rheumatol 1996; 23: 112-19.

11. Salvarani C, Gabriel SE, O'Fallon WM, Hunder GG. The incidence of giant cell arteritis in Olmsted County, Minnesota: Apparent fluctuations in a cyclic pattern. Ann Intern Med 1995; 123: 192-194.

12. V. Petursdottir, H. Johansson, E. Nordborg, C. Nordborg. The epidemiology of biopsy-positive giant cell arteritis: special reference to cyclic fluctuations. Rheumatology 1999; 38: 1208-1212.

13. Machado EBV, Michet CJ, Ballard DJ, Hunder CG, Beard CM, ChuPin $\mathrm{Ch}$ et al. Trends in incidence and clinical presentation of temporal arteritis in Olmsted county, Minnesota, 1950-1985. Arthritis Rheum 1988; 31: 745-749.

14. Baldursson O, Steinsson K, BjornssonJ, Lie JT. Giant cell arteritis in Iceland. An epidemiologic and histopathologic analysis. Arthritis Rheum 1994; 37: 1007-1012.

15. Calvo JM, Magro D, Ramos JL, et al. Arteritis de células gigantes: un estudio descriptivo en el suroeste de España. An Med Interna (Madrid) 2000; 17: 67-70

16. Armona J, Rodríguez-Valverde V, González-Gay MA, Figueroa M, Fernández-Sueiro JL, Blanco R, Martínez-Taboada V. Arteritis de células gigantes. Estudio de 191 pacientes. Med Clin (Barc) 1995; 105: 734-737.

17. Vilalta J, Vilaseca J, Ingelmo M, González L, Tor J, Coca A, Bacardi R, Balcells-Gorina A. Arteritis Temporal. Estudio de 43 casos. Med Clin (Barc) 1982; 78: 307-312.

18. Rivero Puente A, Berasategui Calderón JL, Murie Carrillo de Albornoz JM, Rivero Marcotegui M, García-Bragado Acín F. Arterítis de células gigantes y polimialgia reúmática: diagnóstico y evolución de 90 casos. An Med Interna (Madrid) 2001; 18: 191-194

19. Liu GT, Glaser JS, SchatzNJ, Smith JL. Visual morbidity in giant cell arteritis. Clinical characteristics and prognosis vision. Ophthalmology 1994; 101: 1779-1785

20. Cid M, Font C, Oristrell J, De la Sierra A, Coll-Vinent B, López-Soto A. Vilaseca J, Urbano-Márquez A, Grau JM. Association between strong inflammatory response and low risk of developing visual loss and other cranial isquemic complications in giant cell (temporal) arteritis. Arthritis Rheum 1998; 41: 26-32.

21. González-Gay M, Banco R, Rodríguez- Valverde V, Martínez-Taboada V, Delgado-Rodríguez M, Figueroa M, Uriarte E. Permanent visual loss and cerebrovascular accidents in giant cell arteritis. Arthritis Rheum 1998; 41: 1497-1504.

22. Salvarani C, Cantini F, Boiardi L, Hunder GG. Polymyalgia rheumatica and giant-cell artertis. N Engl J Med 2002; 347: 261-271

23. Liozon E, Herrmann F, Ly K, Robert PY, Loustaud V, Soria P, Vidal E. Risk factors for visual loss in giant cell (temporal) arteritis: a prospective study of 174 patients. Am J Med 2001; 111: 211-7

24. Recommendations for the prevention and treatment of glucocorticoidinduced osteoporosis: 2001 update. American College of Rheumatology Ad Hoc Committee on Glucocoritcoid-Induced Osteoporosis. Arthritis Rheum 2001; 44: 1496-503.

25. Adachi JD, Saag KG, Delmas PD, et al. Two-year effects of alendronate on bone mineral density and vertebral fracture in patients receiving glucocorticoids: a randomized, double-blind, placebo-controlled trial. Arthritis Rheum 2001; 44: 202-1126. 